\title{
DEVELOPMENT OF EXPERIMENTAL PAPER-FEEDING SYSTEM USING CRAWLING-LIKE LOCOMOTION ROBOT
}

\author{
LIN GUO AND FUMIHIKO ASANO \\ School of Information Science, Japan Advanced Institute of Science and Technology, \\ 1-1 Asahidai, Nomi, Ishikawa 923-1292, Japan \\ E-mail: \{guolin,fasano\}@jaist.ac.jp \\ LONGCHUAN LI \\ Research Organization of Science and Technology, Ritsumeikan University, \\ 1-1-1 Nojihigashi, Kusatsu, Shiga 525-8577, Japan \\ E-mail: lltt2144@fc.ritsumei.ac.jp
}

\section{Extended Abstract}

The authors have investigated novel crawling-like locomotion robots that move on slippery downhill and level surface. ${ }^{1,2}$ They numerically showed that high-speed forward sliding locomotion can be generated by positively utilizing the effects of friction force acting on the ground contact point, which is underactuated however indirectly excited by synchronizing the frame rotation via entrainment effect, as well as concentrating the sliding direction via damping effect. ${ }^{3}$ After that, they considered to utilize the robot as an actuator by grounding it via a passive rotational joint as shown in Fig. 1 (a): a simple paper-feeding system. ${ }^{4}$ The wobbling length, $l_{3}[\mathrm{~m}]$, is controlled to follow the desired trajectory, $y_{\mathrm{d}}(t)=A_{m} \sin \left(2 \pi f_{c} t\right)$. Through basic numerical simulations, it was confirmed that the robot's forward motion can be successfully transformed to the paper-feeding motion as shown in Fig. 1 (b). The paper (transported object) model is specified as $m_{\mathrm{p}} \ddot{x}_{\mathrm{p}}=-\mu \lambda_{c}-C_{\mathrm{p}} \dot{x}_{\mathrm{p}}$, and is fed in the negative direction of the $X$-axis by the frictional force generated by the reciprocating motion of the robot body frame. Parametric studies showed that the feeding speed is increased with the increase of the viscosity coefficient in the body frame, $c[\mathrm{~N} \cdot \mathrm{s} / \mathrm{m}]$, but the change of the elastic coefficient, $k[\mathrm{~N} / \mathrm{m}]$, is less effective. It was also confirmed that the feeding speed can be controlled by adjusting the wobbling frequency, $f_{c}[\mathrm{~Hz}]$, and the viscosity coefficient in the paper, $C_{\mathrm{p}}[\mathrm{N} \cdot \mathrm{s} / \mathrm{m}]$.

To confirm the validity of the above numerical results, we developed a prototype paper-

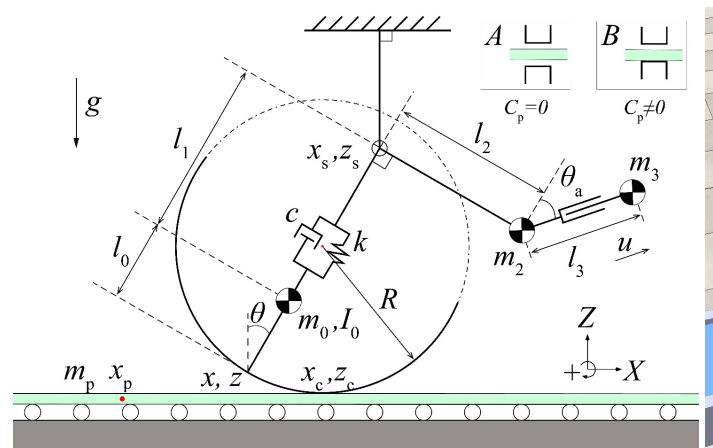

(a)

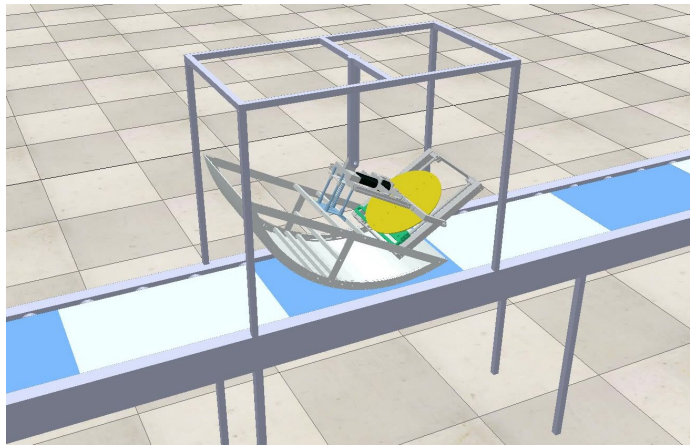

(b)

Fig. 1. Mathematical model and simulation of paper-feeding system using crawling-like locomotion robot 


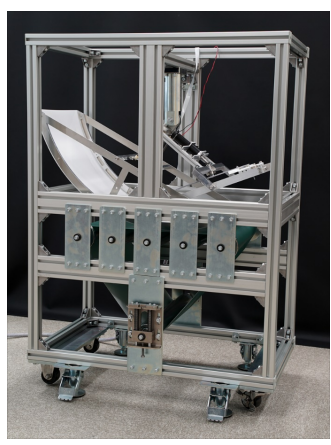

(a)

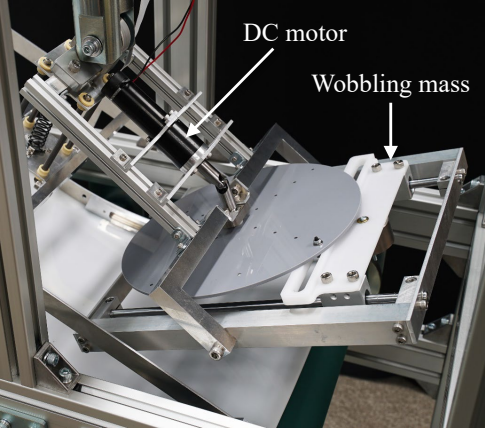

(b)

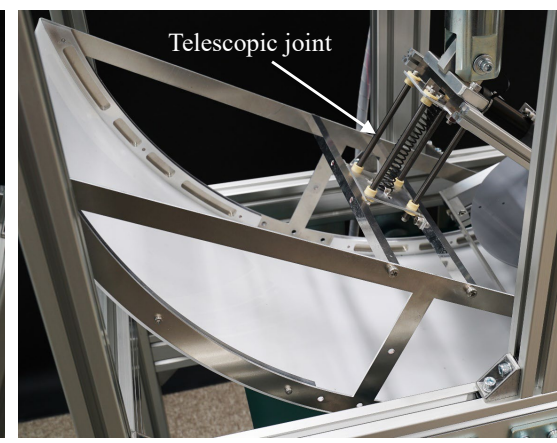

(c)

Fig. 2. Experimental paper-feeding system using crawling-like locomotion robot

feeding system shown in Fig. 2. In this paper, we report some basic experimental results. The crawling-like locomotion robot is composed of two identical arc-shaped frames, a spring mechanism, and an actively-controlled wobbling mass. The bottom plate is made of plastic. It is then possible to reduce the weight and to generate a suitable frictional effect for sliding. The wobbling mass is driven by a DC motor via a slider-crank mechanism. By rotating the motor at a constant speed, the wobbling mass can easily generate an oscillating motion like $y_{\mathrm{d}}(t)$. The weight $m_{3}$ and stroke $A_{m}$ of the wobbling mass can be easily adjusted. The treadmill is passive, and the belt is fed by the robot. The belt tension or feed resistance can be adjusted by using the tension pulleys. We conducted preliminary experiments using this machine, but an ideal operation could not be achieved due to the following problems. The edge of the rail of the spring mechanism (telescopic joint) shown in Fig. 2 (c) caused frictional resistance more than expected, and unexpected oscillation and some other phenomena occurred. For achieving an ideal operation, we are now redesigning some parts. The upper part of the rail is fixed using a set collar. A linear bush is used so that the vertical linear motion operates smoothly. In addition, a rail is set in the spring so that the spring can move in synchronization with the rail connected to the spring body. In the presentation, we will show our latest experimental results.

\section{Acknowledgments}

This research was partially supported by Grant-in-Aid for Scientific Research (C) No. 19K04255, provided by the Japan Society for the Promotion of Science (JSPS). The authors would like to express their gratitude to ONO-DENKI CO., LTD. and GIKEN CO., LTD. for many helpful suggestions and technical supports in development of the experimental crawling-like locomotion robot and treadmill.

\section{References}

1. F. Asano, T. Seino, I. Tokuda and Y. Harata, "A novel locomotion robot that slides and rotates on slippery downhill," Proc. of the IEEE Int. Conf. on Advanced Intelligent Mechatronics, pp. 425-430, 2016.

2. M. Nishihara, T. Seino and F. Asano, "Motion analysis of underactuated locomotion robot with quick return linkages on slippery level surface," Proc. of the IEEE Int. Conf. on Advanced Intelligent Mechatronics, pp. 1663-1668, 2017.

3. L. Li, F. Asano and I. Tokuda, "High-speed sliding locomotion generation on slippery surface of an indirectly controlled robot with viscoelastic body," IEEE Robotics and Automation Letters, Vol. 4, Iss. 3, pp. 2950-2957, 2019.

4. L. Guo, L. Li and F. Asano, "Conveyor manipulation system using high-speed crawling-like locomotion robot," Proc. of the SWARM2019: The 3rd Int. Symp. on Swarm Behavior and Bio-Inspired Robotics, pp. 258-261, 2019. 